\title{
Finance, Markets and Valuation
}

\author{
Estrategia de expansión internacional en el sector \\ bancario español: El ejemplo del BBVA
}

\section{Internationalization strategy in the spanish banking sector: The BBVA case}

\section{Alejandra Albero ${ }^{1}$}

${ }^{1}$ Facultad de Administración y Dirección de Empresas, Universidad Politécnica de Valencia. Valencia, España.Email: alalval@upv.es

JEL: F23; G15; G21

\section{Resumen}

En un contexto en el que se prima la globalización económica, la expansión internacional de las grandes entidades financieras se muestra como un factor básico para promover la integración de los sistemas bancarios a nivel mundial. Es por ello que resulta de gran utilidad conocer los factores que influyen en la internacionalización bancaria, a nivel interno y a nivel externo. Este trabajo describe la expansión del Banco BBVA, distinguiendo tres etapas, y el resultado obtenido con esta estrategia de diversificación de la presencia internacional.

El proceso muestra factores cómo la productividad o la desregulación financiera determinan las estrategias a seguir. Por último, se analiza cómo la diversificación de la actividad financiera permite obtener nuevas fuentes de beneficios, que amortiguan las crisis financieras en los mercados nacionales e internacionales.

Keywords: Internacionalización bancaria; Desregulación financiera; Globalización.

DOI:

10.46503/WYGE6651

Corresponding author Alejandra Albero

Recibido: 13 Sep 2018 Revisado: 16 Oct 2018 Aceptado: 17 Oct 2018

Finance, Markets and Valuation

ISSN 2530-3163.

\begin{abstract}
In the present economic context which fosters economic globalization, the internationalization of the big financial institutions is a key factor to increase the integration of the different banking systems worldwide. For this reason, it is important to study which variables, both internal and external, influence the internationalization process. This paper describes the expansion strategy of the Spanish bank BBVA, where three stages are identified. The outcomes of this process is analysed, as well. The study shows how factors such as productivity and financial deregulation are determinant is the strategy's selection. Furthermore, the diversification stage reached makes it possible to obtain new benefit sources and reduce the impact of financial crisis in both national and international markets.
\end{abstract}

Keywords: Internationalization in the banking sector; Deregulation; Globalization.

Cómo citar este artículo: Albero, A. (2018) Estrategia de expansión internacional en el sector bancario 


\section{Introducción}

En las últimas décadas, el sistema financiero internacional ha experimentado un proceso de integración sin precedentes. Esta situación es de gran importancia económica, habida cuenta del impacto que tiene el sector financiero sobre el crecimiento económico. Así, el proceso de integración financiera global que se fomenta con decisión desde instancias internacionales tiene una profunda influencia en la economía, pudiendo ser esta influencia tanto positiva como negativa.

Dentro de este contexto cabe enmarcar la creación de grandes conglomerados financieros con presencia internacional, entre los que destacan los grandes bancos. Dada la importancia del tema, la internacionalización de los bancos es una problemática que se ha estudiado profusamente, desde diversas perspectivas y en diferentes áreas geográficas a lo largo de las últimas décadas.

Este trabajo se va a centrar, a modo de estudio del caso, en el proceso de expansión seguido por el banco español BBVA.

El resto del trabajo se estructura de la siguiente manera. A continuación, la sección dos revisa la principal literatura acerca de los procesos de internacionalización bancaria, tanto a nivel español como de otras entidades financieras. La sección tres explica el proceso seguido por BBVA desde sus inicios, en 1857, momento en el que nace la entidad, hasta la actualidad, presentando una descripción cuantitativa desde el año 2005. De esta forma, se comprueba cuáles son actualmente las regiones y los países en los cuales el BBVA tiene presencia física y las principales variables económicas que permiten cuantificar dicha presencia, tales como número de créditos por zonas geográficas, o beneficios obtenidos por la diversificación del negocio a nivel internacional. La sección cuarta se dedica a analizar el impacto de la estrategia de internacionalización seguida por el BBVA en el beneficio consolidado de la entidad. Finalmente, la sección quinta presenta las principales conclusiones del trabajo.

\section{El proceso de internacionalización de la banca}

Conocer las características de los bancos que emprenden el proceso de expansión internacional y qué estrategias siguen ha sido objeto de gran número de estudios.

Diversos estudios han dedicado su trabajo al desarrollo de modelos teóricos que expliquen el comportamiento de la banca en su proceso de internacionalización, estableciendo los factores que influyen en las decisiones estratégicas de internacionalización, como por ejemplo el modelo econométrico de González Expósito (2004) que tiene por objeto la comprobación empírica de la influencia de factores ligados a las economías de los países en los que se ha localizado la banca española sobre la inversión directa extranjera de nuestras entidades. Por otra parte, en el modelo de selección de Buch, Koch, y Koetter (2014) se analizan las diferencias de productividad entre empresas para concluir que esas diferencias pueden determinar la actividad internacional de las firmas no financieras.

En el modelo llamado IP de Lee, Song, y Kwak (2014) se analiza el efecto de la crisis en la internacionalización de los bancos desde la perspectiva de la teoría de redes. Se define empleando el modelo del proceso de internacionalización, donde se muestra en particular el papel del aprendizaje y el compromiso en la expansión al extranjero, con servicios bancarios en condiciones estables y en períodos críticos.

Algunos trabajos concluyen que la heterogeneidad en el tamaño y la productividad son centrales. En los modelos de intercambio, Buch, Koch, y Koetter (2011) analizan las características de las empresas exportadoras. Este estudio presenta evidencia descriptiva de una heterogeneidad 
similar entre los bancos internacionales como proveedores de servicios financieros.

La dinámica e intensidad de la actividad internacional diferencia entre dos tipos (Sierra Fernández, 2007): la oportunista, estrategia en la que la implantación se realiza de una vez, y la gradual, en la que la expansión se realiza de forma exploratoria y secuencial, a medida que se incrementa la experiencia y su presencia en el país de destino de su inversión.

En cuanto al modo de entrada utilizado en el crecimiento internacional, se diferencian dos modelos, basados en un crecimiento interno o externo. En el primer modelo, la entidad bancaria implanta una filial propia, mientras que el segundo modelo crece mediante alianzas externas con otras entidades nacionales.

La resiliencia de las entidades financieras para adaptarse y recuperarse de los efectos de una crisis económica que ha afectado gravemente su negocio y sus resultados, es un aspecto que ha sido objeto de diversos estudios. En el análisis de Markman y Venzin (2014), se desarrolla una medida para la resiliencia llamada "Volare" para comparar las entidades financieras más resilientes, con las menos resilientes. Esta medida identifica los factores diferenciales en las entidades financieras que se internacionalizan, tales como el tamaño del banco, la solidez del mercado propio y la complejidad del producto y del mercado.

Tratando los determinantes que mueven a los bancos a salir de su zona geográfica tradicional, observamos el análisis realizado por Hernando, Nieto, y Wall (2009), que centra su investigación en los factores que influyen en las adquisiciones bancarias, dentro del país y entre los países de la UE, hasta 2004. Las conclusiones del trabajo muestran, que las entidades bancarias, han generado costes superiores a sus beneficios, y que las entidades de mayor volumen, tienden a ser adquiridas por otras entidades dentro del mismo país. Por otra parte, los bancos que operan en zonas geográficas de mayor concentración, tienen menos posibilidades de ser adquiridos por otra entidad nacional, pero más de ser adquiridos por otros bancos de la Unión Europea.

Para definir la influencia de los ciclos económicos en las decisiones de internacionalización bancaria, podemos destacar el modelo econométrico propuesto por Choudhry y Jayasekera (2012), que muestra el efecto asimétrico de las buenas o malas noticias dentro de un contexto económico. Se asignan betas variables en el tiempo, que se crean mediante el modelo bivariado BEKK GARCH, y se genera un modelo que aclara el nivel de eficiencia del mercado y las estrategias de protección.

Por último, observamos otra investigación que focaliza su análisis en la productividad de las entidades que emprenden procesos de internacionalización (Buch y cols., 2014) que analiza la formas de entrada, bien mediante adquisición o establecimiento de filiales en el extranjero. Se concluye que las entidades financieras más productivas tienen mayor probabilidad de entrada en mercados complejos, que tienen un mayor nivel de activos en el extranjero, y por otra parte concluye que un mayor nivel de riesgo asumido hace que la entrada sea menos probable.

Podemos concluir que la literatura publicada, pone de manifiesto que las entidades financieras con mayor productividad y eficiencia, son las más propensas a expandir su negocio a nivel internacional, aprovechando las nuevas tecnologías, para diversificar su actividad, amortiguar los ciclos económicos y crecer.

Para seguir estudiando el fenómeno de la internacionalización bancaria, desde el punto de vista de la expansión española, seguidamente se realiza una breve revisión de la literatura publicada sobre los determinantes de la internacionalización en el caso de la banca española. 


\subsection{Internacionalización de la banca española}

En cuanto a la literatura publicada en referencia a la internacionalización de la banca española, destaca la investigación de la estrategia internacional de los principales bancos del país, las formas de entrada en el exterior, la relación entre el nivel de internacionalización y la rentabilidad de las entidades, tal y como comenta Sánchez Peinado (2001). Paralelamente, se ha analizado la influencia de los factores vinculados a las características de los países en los que se ha realizado la inversión (González Expósito, 2004), empleándose variables tales como la inversión directa en el extranjero realizada por el sector bancario y el resto de sectores económicos, el tamaño del mercado destino, el nivel de bancarización, la evolución de los tipos de interés, el tipo de cambio, o el volumen de comercio exterior entre España y el país de destino.

Es importante analizar las razones por las cuales las entidades financieras españolas deciden dirigir su inversión principalmente hacia Latinoamérica. El trabajo de Rodríguez Inciarte (2004) estudia este comportamiento mediante datos descriptivos, teniendo en cuenta los efectos de la globalización sobre el sistema financiero. Posteriormente publica otro análisis vinculado al anterior Rodríguez Inciarte (2005) cuya base se centra en el incremento de las tecnologías de la información y el nexo de unión entre los mercados, junto con el marco político y económico que fuerza la adaptación a nuevos mercados y contextos.

En la búsqueda de nuevos mercados para diversificar su actividad, Gil (2005) estudia el desarrollo del negocio, la reducción de márgenes y el incremento de la competencia, llegando a la conclusión de que la adquisición de bancos en Latinoamérica se debe a la posibilidad de aprovechar las ventajas que presentan esos mercados bancarios menos desarrollados, exportando los bancos españoles sus plataformas tecnológicas y su know how.

Por otra parte, según Goddard, Molyneux, Wilson, y Tavakoli (2007), se deben tener en cuenta las barreras que todavía persisten para la total integración de la banca, sobre todo en lo referente a la banca minorista. Estos autores analizan las relaciones prestatario-prestamista a largo plazo, la estructura de propiedad, el cambio tecnológico y la eficiencia bancaria, en un contexto de mercados financieros altamente competitivos.

Es importante destacar la cautela de los grandes bancos españoles a la hora de internacionalizarse, tal y como señala Sierra Fernández (2007), quien muestra que las entidades financieras aplican estrategias defensivas y reducidos volúmenes de recursos y riesgos en un primer momento, para posteriormente incrementar la intensidad y la agresividad. Así, a finales de 2007 los bancos españoles llegan a liderar diferentes servicios bancarios y parabancarios en grandes países como Brasil y México.

\section{Orígenes y expansión internacional del banco BBVA}

En este epígrafe se describe cuál ha sido la estrategia de expansión desarrollada concretamente por el BBVA, desde sus orígenes hasta el presente (BBVA, 2018).

El BBVA es el resultado de una serie de integraciones bancarias. De entre las entidades financieras que se han ido incorporando destacan el Banco de Bilbao, el Banco de Vizcaya y Argentaria, que fue una sociedad estatal con estatuto de banco que aglutinaba entidades financieras estatales como la Caja Postal o el Banco Exterior.

El Banco de Bilbao, el más antiguo de todos, se crea en 1857 en un entorno de crecimiento económico. En sus inicios se dedicó a financiar al sector siderúrgico e infraestructuras relevantes. Por su parte, el Banco de Vizcaya se funda en 1901 y la Caja Postal, entidad de derecho público, empieza a operar en 1916. 
A partir de 1960, el Banco de Bilbao comienza a absorber a otros bancos, mientras que el Banco de Vizacaya también crece y amplía su red comercial.

Un hito fundamental en la historia de BBVA es la fusión del Banco de Bilbao y el Banco de Vizcaya para crear el BBV, en 1988.

Once años más tarde, se produce la incorporación del grupo Argentaria, creándose el banco BBVA. El nuevo banco goza de un tamaño relevante, gran solvencia y mucho potencial de crecimiento.

A principios del año 2000, cambia su marca y se denomina BBVA, lo que ayuda a posicionarse en el mercado como una marca sólida y diferenciada, que ofrece seguridad y rentabilidad. Con la nueva red de oficinas, formada por todas las entidades que se han integrado, el BBVA se desarrolla a nivel nacional, lo que incrementa su dimensión y diversifica sus riesgos, redundando en un incremento de beneficios. Algunas publicaciones lo nombran el mejor banco del mundo (Forbes) y de España (The Banker) en el año 2000, mejor banco de Latinoamérica (Forbes) y mejor banco europeo (Lafferty) en 2001.

Más recientemente, en 2013, BBVA terminó el proceso de incorporación al grupo de Unnim Banc, que agrupaba diversas antiguas Cajas de Ahorro, y fue adquirido por BBVA, fortaleciendo más aún su posición dentro del mercado nacional.

En cuanto a la expansión internacional del BBVA, cabe destacar el fuerte proceso de expansión experimentado desde 1995, tras haber alcanzado una posición dominante en el mercado español.

El BBVA inicia su proceso de internacionalización en América Latina, área en la que se presenciaba un bajo nivel de desarrollo económico, junto con niveles de eficiencia y productividad reducidos. La proximidad cultural fue el factor decisivo por el cual la entidad se implantó en primer lugar en América Latina, ya que facilitaba la distribución de sus productos financieros y la rápida adaptación al nuevo entorno social y económico. Así, entre 1995 y 2000 adquiere bancos en Perú, México, Colombia, Argentina, Venezuela, Puerto Rico, Brasil y Chile.

A partir de 2004 se lanza a por otros mercados, especialmente el de EEUU y el de China, sin dejar de crecer en Iberoamérica.

Tras la crisis financiera y económica de 2008, se producen desinversiones en todas las áreas geográficas, pero manteniendo siempre una fuerte presencia internacional. Así, en 2018 tiene presencia en más de 30 países, en los que tiene más de 75 millones de clientes y gestiona más de 690 mil millones de euros en activos y más de 131 mil empleados.

\section{Grado de internacionalización del BBVA}

La tabla 1 muestra la presencia internacional del grupo BBVA desde 2005 hasta 2016. Se observa claramente cómo a lo largo de todo el periodo el banco ha estado presente en los principales mercados mundiales, si bien la presencia en países concretos ha variado con motivo de las fluctuaciones económicas y crisis internacionales. 
Finance, Markets and Valuation Vol. 4, Num. 2 (Julio-Diciembre 2018), 23-33

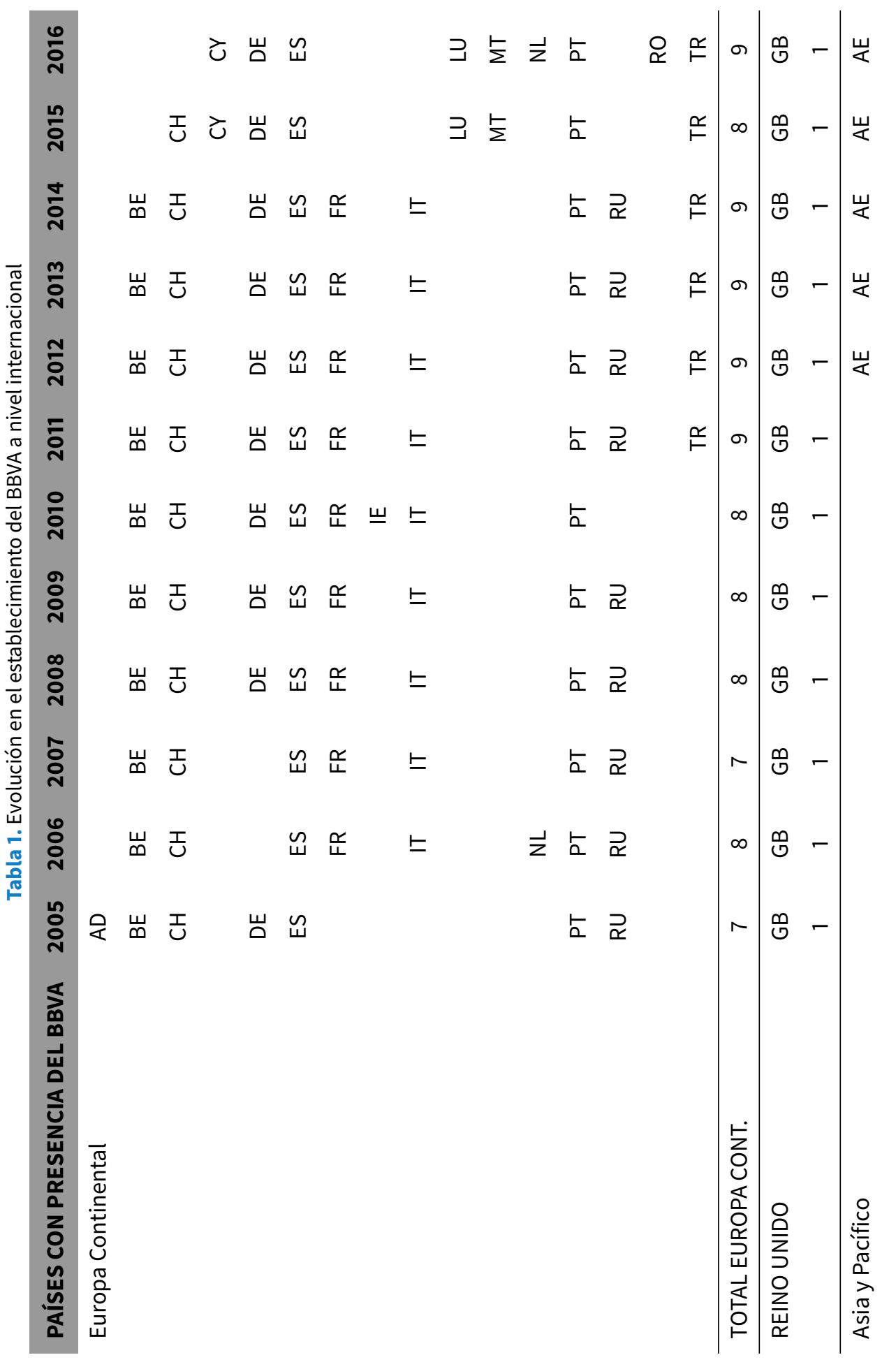


Finance, Markets and Valuation Vol. 4, Num. 2 (Julio-Diciembre 2018), 23-33

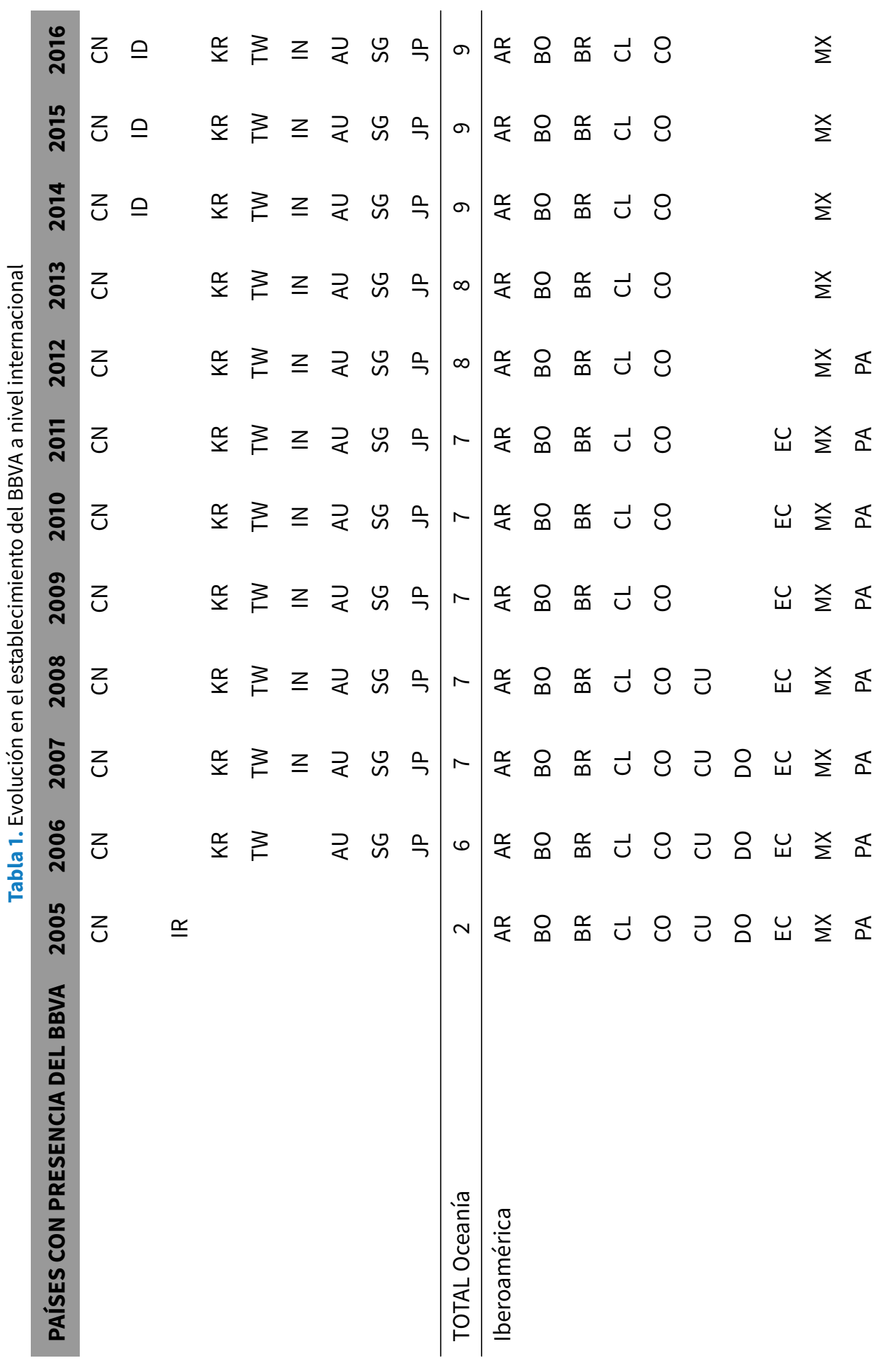


Finance, Markets and Valuation Vol. 4, Num. 2 (Julio-Diciembre 2018), 23-33

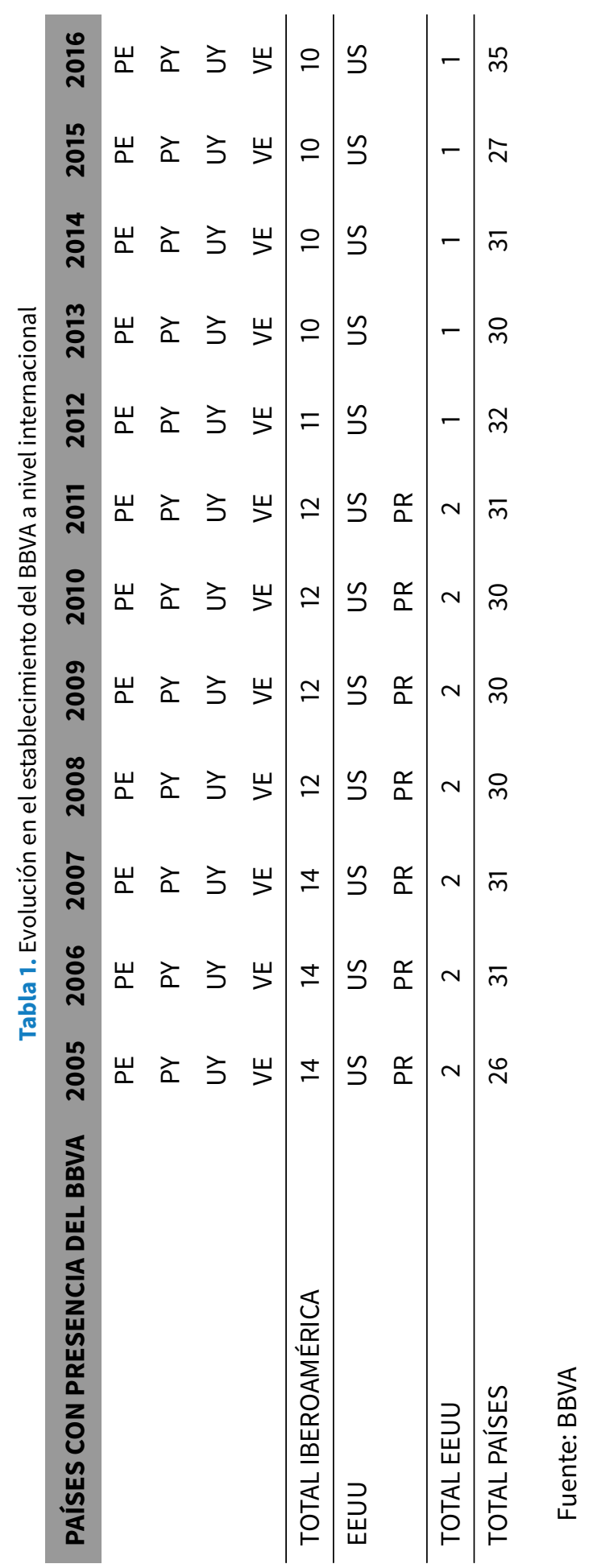


Finance, Markets and Valuation Vol. 4, Num. 2 (Julio-Diciembre 2018), 23-33

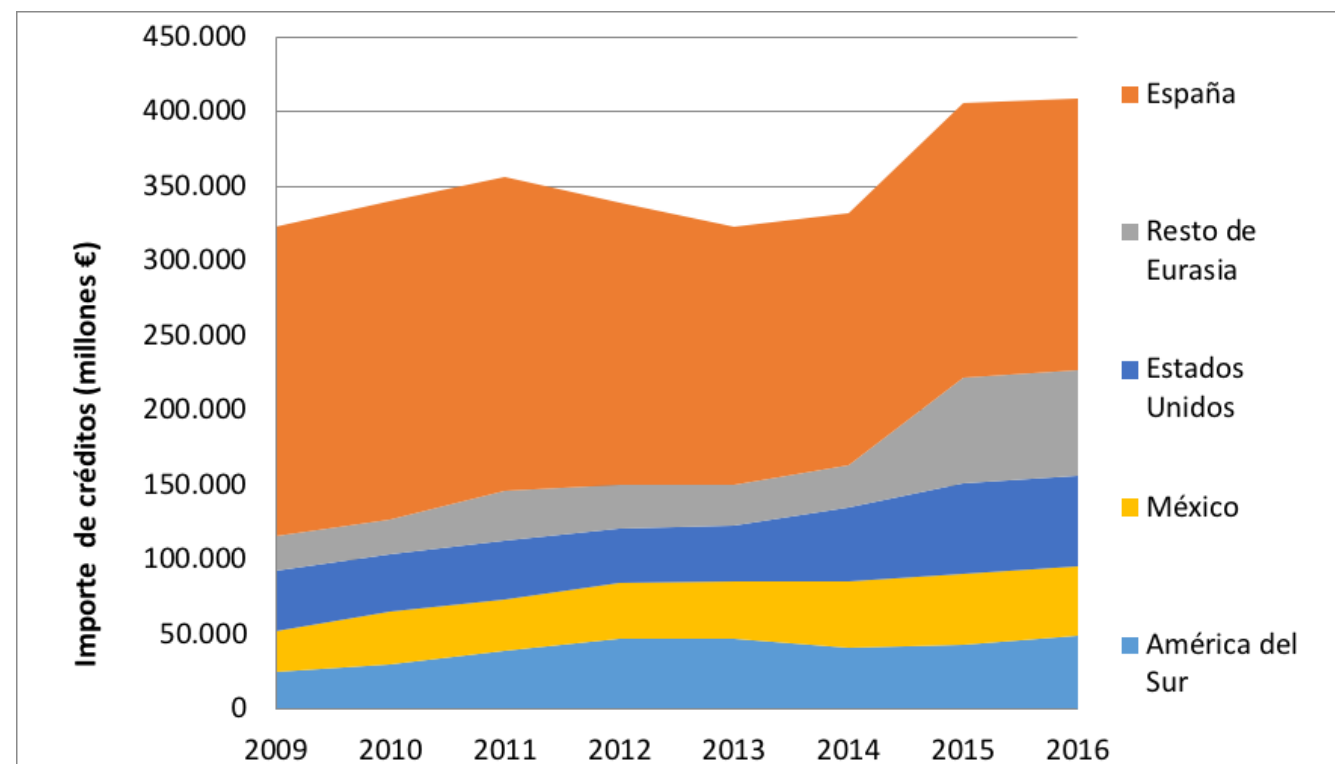

Figura 1. Número de créditos concedidos en principales países de inversión

Fuente: BBVA

Obviamente, el BBVA no tiene el mismo grado de implantación en todos los países en los que opera. Así, la figura 1 muestra la importancia de tres países concretos, España, EEUU y México, en términos de importe de créditos concedidos.

\section{Resultado de la estrategia de diversificación y presencia internacional}

Dos de los objetivos principales de las estrategias de expansión geográfica de las empresas es aumentar ingresos y el beneficio consolidado, al tiempo que se reduce el riesgo, es decir, la volatilidad de los beneficios originada por fluctuaciones en el ciclo económico en los diferentes mercados, de forma que disminuciones del beneficio en un mercado se ven compensadas por mayores beneficios en otras áreas geográficas.

En el caso de BBVA, la expansión internacional ha contribuido decididamente al beneficio de la entidad, como vemos en la figura 2.

La figura muestra claramente cómo se ha modificado la estrutura geográfica de los beneficos entre el año 2009 y el 2016. Efectivamente, se observa inequívocamente que el peso absoluto y relativo del mercado español ha disminuido considerablemente. Es decir, de no haberse implementado la estrategia de internacionalización, los beneficios de la entidad se hubieran reducido dramáticamente.

En cuanto a la aportación de cada una de las zonas geográficas al beneficio consolidado de BBVA, se pone de relevancia que en 2016 México es el país que más aporta, seguido de España y EEUU. El resto de mercados desempeña un papel mucho más residual.

\section{Conclusiones}

En este artículo se ha analizado la evolución internacional del BBVA, elegido por la estrategia de expansión geográfica seguida en las últimas dos décadas que han hecho del banco uno de los principales actores en la escena internacional.

En primer lugar, se han presentado los diversos enfoques teóricos y los estudios que analizan 
Finance, Markets and Valuation Vol. 4, Num. 2 (Julio-Diciembre 2018), 23-33

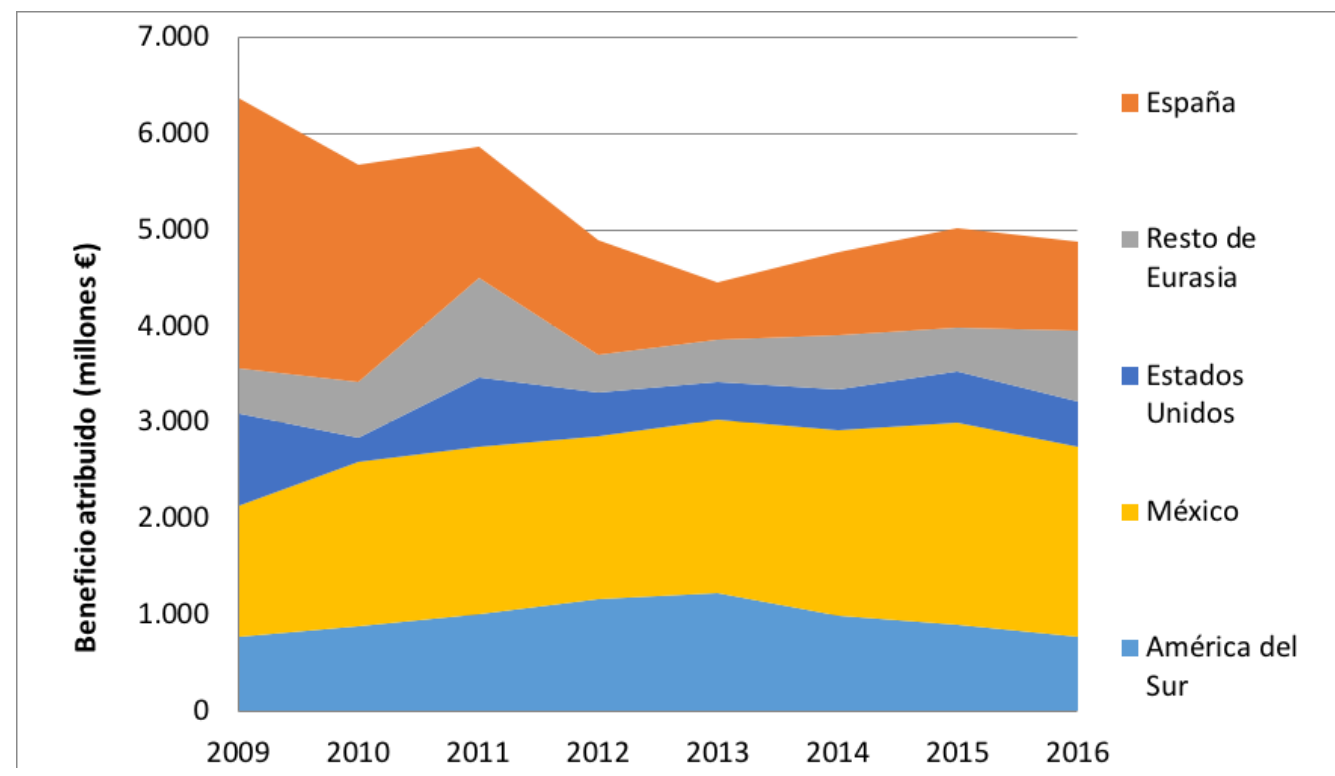

Figura 2. Beneficio atribuido en principales países de inversión

Fuente: BBVA

las estrategias seguidas por las entidades bancarias en su proceso de internacionalización. Dentro de esta temática, se han tratado aspectos tales como las motivaciones para dar el salto a los mercados extranjeros, las fases a seguir en ese proceso o estrategia de localización. Otras cuestiones que han llamado la atención de los expertos son las características que reúnen los bancos que alcanzan el éxito internacional y la importancia de factores externos como el proceso de desregulación a nivel mundial, la globalización económica y financiera o los avances tecnológicos.

A continuación, el estudio se centra en el BBVA, presentando la historia de su estrategia de expansión internacional y los resultados alcanzados en la actualidad gracias a esta estrategia. La internacionalización bancaria de la entidad ha mitigado los efectos de la crisis económica en sus balances y especialmente en el beneficio obtenido, viéndose afectado en menor medida que el resto de entidades españolas menos enfocadas al exterior. Efectivamente, hemos comprobado que el Banco BBVA cuenta con una presencia muy asentada en el mercado mexicano y estadounidense.

\section{Referencias}

BBVA. (2018). Información corpativa (Inf. Téc.). Banco Bilbao Vizcaya Argentaria (BBVA). Descargado de https://www.bbva.com/es/informacion-corporativa/historia-de -bbva/ (Consultado el 15/05/2018)

Buch, C. M., Koch, C. T., y Koetter, M. (2011). Size, productivity, and international banking. Journal of International Economics, 85(2), 329-334. doi: https://doi.org/10.1016/j.jinteco.2011.07.001

Buch, C. M., Koch, C. T., y Koetter, M. (2014). Should i stay or should i go? bank productivity and internationalization decisions. Journal of Banking \& Finance, 42, 266-282. doi: https://doi.org/10.1016/j.jbankfin.2014.02.003

Choudhry, T., y Jayasekera, R. (2012). Comparison of efficiency characteristics between the 
banking sectors of US and UK during the global financial crisis of 2007-2011. International Review of Financial Analysis, 25, 106-116. doi: https://doi.org/10.1016/j.irfa.2012.09.002

Gil, G. (2005). Internacionalización de la banca española: la empresa española ante los nuevos retos de la internacionalización. En Banco de España (Ed.), La empresa española ante los nuevos retos de la internacionalización (pp. 103-114). APIE.

Goddard, J., Molyneux, P., Wilson, J. O., y Tavakoli, M. (2007). European banking: An overview. Journal of Banking \& Finance, 31(7), 1911-1935. doi: https://doi.org/10.1016/j.jbankfin.2007.01.002

González Expósito, I. (2004). La inversión directa de la banca española en el exterior: Factores determinantes y estrategias. Boletín Económico de ICE, 817, 103-114.

Hernando, I., Nieto, M. J., y Wall, L. D. (2009). Determinants of domestic and cross-border bank acquisitions in the european union. Journal of Banking \& Finance, 33(6), 1022-1032. doi: https://doi.org/10.1016/j.jbankfin.2008.10.017

Lee, J.-W., Song, H. S., y Kwak, J. (2014). Internationalization of korean banks during crises: The network view of learning and commitment. International Business Review, 23(6), 1040-1048. doi: https://doi.org/10.1016/j.ibusrev.2014.06.009

Markman, G. M., y Venzin, M. (2014). Resilience: Lessons from banks that have braved the economic crisis-and from those that have not. International Business Review, 23(6), 1096-1107. doi: https://doi.org/10.1016/j.ibusrev.2014.06.013

Rodríguez Inciarte, M. (2004). La expansión exterior de la banca española. Universia Business Review(3), 12-25.

Rodríguez Inciarte, M. (2005). Los retos de la economía española en la actualidad: la banca española ante la globalización. Información Comercial Española, ICE: Revista de economía(826), 313-322.

Sánchez Peinado, E. (2001). Internacionalización de la banca española: evolución y resultados en los años noventa. Información Comercial Española, ICE: Revista de economía(794), 137-162.

Sierra Fernández, M. P. (2007). Estrategias de internacionalización de la gran banca española. Pecvnia(5), 229-272. doi: https://doi.org/10.18002/pec.v0i5.717 